Pacific Journal of Mathematic 


\title{
EXISTENCE OF TRICONNECTED GRAPHS WITH PRESCRIBED DEGREES
}

\author{
S. B. RAO AND A. RAMAChandRa RaO
}

Necessary and sufficient conditions for the existence of a $p$-connected (linear undirected) graph with prescribed degrees $d_{1}, d_{2}, \cdots, d_{n}$ are known for $p=1,2$. In this paper we solve this problem for $p=3$.

Let $d_{1}, d_{2}, \cdots, d_{n}$ be positive integers and let $d_{1} \leqq d_{2} \leqq \cdots \leqq d_{n}$.

Lemma. If a triconnected graph $G$ exists with degrees $d_{1}, d_{2}, \cdots$, $d_{n}$, then

(1) $\quad d_{i} \geqq 3$.

(2) $d_{1}, d_{2}, \cdots, d_{n}$ is graphical, i.e., there exists a graph with these degrees.

(3) $d_{n}+d_{n-1} \leqq m-n+4$ where $2 m=\sum_{i=1}^{n} d_{i}$.

(4) If $d_{n}+d_{n-1}=m-n+4$, then $m \geqq 2 n-2$.

Proof. (1) and (2) are evident. To prove (3), let $x_{n}, x_{n-1}$ be the vertices of $G$ with degrees $d_{n}$ and $d_{n-1}$ respectively. Then the number of edges in $G-\left\{x_{n}, x_{n-1}\right\}$ is $m-\left(d_{n}+d_{n-1}-1\right)$ or $m-\left(d_{n}+d_{n-1}\right)$ according as $x_{n}, x_{n-1}$ are adjacent or not adjacent in $G$. Also $G-\left\{x_{n}\right.$, $\left.x_{n-1}\right\}$ is connected, so (3) follows. If now $d_{n}+d_{n-1}=m-n+4$, then

$$
2 m \geqq d_{n}+d_{n-1}+3(n-2)=m+2 n-2 .
$$

This completes the proof of the lemma.

THEOREM. Conditions (1) to (4) of the lemma are necessary and sufficient for the existence of a triconnected graph with degrees $d_{1}, d_{2}, \cdots, d_{n}$.

Proof. Necessity was proved in the lemma.

To prove sufficiency, first let conditions (1), (3) be satisfied and let $d_{n}+d_{n-1}=m-n+4=n+\lambda$ where $2 \leqq \lambda \leqq n-2$. Let $k$ be the number of $d_{i}$ such that $1 \leqq i \leqq n-2$ and $d_{i}=3$. Then define

$$
e_{i}=d_{i}-2 \text { for } i=k+1, \cdots, n-2 \text {. }
$$

Then we have

$$
\begin{aligned}
& \sum_{i=1}^{n-2} d_{i}=2 m-d_{n}-d_{n-1}=3 n+\lambda-8, \\
& \sum_{i=k+1}^{n-2} e_{i}=3 n+\lambda-8-3 k-2(n-2-k)=n+\lambda-k-4 .
\end{aligned}
$$


Define now $\eta=n-2-\lambda$ and $\varepsilon=k-\eta$. Then $\eta \geqq 0$, and $\varepsilon \geqq 2$ since

$$
\begin{aligned}
2 m & \geqq m-n+4+3 k+4(n-2-k) \\
& =m+3 n-k-4
\end{aligned}
$$

and so

$$
\lambda=m-2 n+4 \geqq n-k .
$$

Write now

$$
e_{i}= \begin{cases}1 & \text { for } i=1,2, \cdots, \varepsilon, \\ 2 & \text { for } i=\varepsilon+1, \cdots, k, \\ d_{i}-2 & \text { for } i=k+1, \cdots, n-2 .\end{cases}
$$

Then $\sum_{i=1}^{n-2} e_{i}=2(n-3)$ and so there exists a tree $T$ with degrees $e_{1}, \cdots e_{n-2}$, attained by the vertices $x_{1}, \cdots, x_{n-2}$, say, in that order [2]. Take two more vertices $x_{n-1}$ and $x_{n}$ and join them. Also join each of $x_{n-1}, x_{n}$ to $x_{i}$ for $i=1, \cdots, \varepsilon, k+1, \cdots, n-2$. Of the $\eta$ vertices $x_{\varepsilon+1}, \cdots, x_{k}$, join $d_{n-1}-1-\varepsilon-n+2+k$ to $x_{n-1}$ and the rest $\left(d_{n}-1-\varepsilon-n+2+k\right.$ in number $)$ to $x_{n}$. Note that

$$
d_{n-1}-1-\varepsilon-n+2+k=d_{n-1}-\lambda-1 \geqq 0 .
$$

The graph we thus obtain has degrees $d_{1}, \cdots, d_{n}$ and is triconnected since any vertex of $T$ with degree in $T$ less than 3 is joined to either $x_{n-1}$ or $x_{n}$.

Next let conditions (1), (2) be satisfied and let

$$
d_{n}+d_{n-1} \leqq m-n+3 \text {. }
$$

Then $d_{n}<m-n+2$, so there exists a biconnected graph $G$ with degrees $d_{1}, d_{2}, \cdots, d_{n}$ [2]. If $G$ is not triconnected, let $x_{i}, x_{j}$ be two vertices such that $G-\left\{x_{i}, x_{j}\right\}$ is disconnected. Let $C_{1}, C_{2}, \cdots$ be the components of $G-\left\{x_{i}, x_{j}\right\}$. By (1), $\left|C_{g}\right| \geqq 2$ for $g=1,2, \cdots$. Also by hypothesis,

$$
m-d_{i}-d_{j} \geqq n-3,
$$

so it follows that one of the components, say $C_{1}$, contains a cycle.

We first prove that there exists an edge $(x, y)$ in $C_{1}$ and two chains $\mu_{1}, \mu_{1}^{\prime}$ of $G$ connecting $x$ and $y$ such that $(x, y), \mu_{1}, \mu_{1}^{\prime}$ are disjoint except for $x$ and $y$, and $\mu_{1}$ is contained in $C_{1}$. Since $G$ is biconnected, there exists a chain connecting $x_{i}$ and $x_{j}$ with all intermediate vertices in $C_{2}$.

If now two vertices $x, y$ with degree two in $C_{1}$ are adjacent and belong to a cycle of $C_{1}$, the required edge is $(x, y)$. So we may take 
that no two vertices of degree two in $C_{1}$ can belong to a block (on more than two vertices) and be adjacent. Let $B$ be any block of $C_{1}$ which is not an edge. If some cycle of $B$ has a chord $(x, y)$, then $(x, y)$ is the required edge. Otherwise, by the results of [1], two vertices $y, z$ of degree two in $B$ will be adjacent to a vertex $x$ of degree three in $B$. If $w$ is another vertex of $B$ adjacent to $x$, then there is a chain connecting $w$ to $y$ in $B-\{x\}$. This chain together with $(x, w)$ may be taken as $\mu_{1}$. To get $\mu_{1}^{\prime}$, go from $x$ to $z$ along $(x, z)$, from $z$ to $x_{i}$ or $x_{j}$ (through another block of $C_{1}$ at $z$ if necessary), then to $y$. Thus $(x, y)$ is the required edge.

Let now $(x, y)$ be an edge of $C_{1}$ chosen as explained above. If $C_{2}$ is a tree, take any edge $(u, v)$ of $C_{2}$. Then $(u, v)$ is a chord of a cycle of $G$. If $C_{2}$ is not a tree, choose an edge $(u, v)$ of $C_{2}$ such that there are chains $\mu_{2}, \mu_{2}^{\prime}$ of $G$ connecting $u$ and $v,(u, v), \mu_{2}, \mu_{2}^{\prime}$ are disjoint except for $u, v$, and $\mu_{2}$ is contained in $C_{2}$.

We define $f_{G}(s, t)$ to be the number of components of $G-\{s, t\}$. Now we will make a modification on $G$ so that the degrees of the vertices are unaltered, $f\left(x_{i}, x_{j}\right)$ decreases and $f(s, t)$ does not increase for any two vertices $s$ and $t$.

First we associate with $x$, a subset $A(x)$ of $\left\{x_{i}, x_{j}\right\}$ by the following rule. $x_{i} \in A(x)$ if and only if there is a chain $\nu$ connecting $x$ to $x_{i}$ with all intermediate vertices in $C_{1}$ such that $\nu$ is disjoint with $(x, y)$ and $\mu_{1}$ except for $x$. Similarly $A(y)$ is defined. If $C_{2}$ is a tree, put $A(u)=A(v)=\left\{x_{i}, x_{j}\right\}$. Otherwise $A(u), A(v)$ are defined in a manner similar to that of $A(x)$ and $A(y)$. Now $A(x), A(y)$ are made nonempty by a proper choice of $\mu_{1}$, and $A(u), A(v)$ are made nonempty by a proper choice of $\mu_{2}$ (in case $C_{2}$ is not a tree).

Now suppress the edges $(x, y),(u, v)$ and join $x$ to one of $u, v$ and $y$ to the other as follows. Join $x$ to $u$ if $A(x) \neq A(u)$ and $A(y) \neq A(v)$ whenever such a choice is possible. Let the new graph thus obtained be $H$. To be specific we take that $x$ is joined to $u$ in $H$.

First we show that $H$ is biconnected. Obviously $G_{1}=G-(x, y)$ is biconnected. Now we show that $(u, v)$ is a chord of a cycle of $G_{1}$. If $C_{2}$ is a tree, then the cycle is

$$
(u, x)+\mu_{1}[x, y]+(y, v)+\left[v, \cdots, p_{1}\right]+\left(p_{1}, x_{i}\right)+\left(x_{i}, p_{2}\right)+\left[p_{2}, \cdots, u\right]
$$

where $p_{1}, p_{2}$ are suitable pendant vertices of $C_{2}$. Otherwise the cycle is

$$
\mu_{2}[u, v]+\mu_{2}^{\prime}[v, u]
$$

where if $\mu_{2}^{\prime}$ contains the edge $(x, y)$, then $(x, y)$ is replaced by $\mu_{1}[x, y]$ and the resulting cycle is made elementary.

Trivially now $f_{G}\left(x_{i}, x_{j}\right)=f_{H}\left(x_{i}, x_{j}\right)+1$. Next we will show that 


$$
f_{G}(s, t) \geqq f_{H}(s, t)
$$

for any two vertices $s$ and $t$. For this it is enough to show that $x, y$ are connected and $u, v$ are connected in $H-\{s, t\}$.

First let $s=x_{i}$. Now $x, y, u, v$ belong to a cycle in $H-\left\{x_{i}\right\}$, so (5) follows. So we may take $\{s, t\} \cap\left\{x_{i}, x_{j}\right\}=\varnothing$.

Now let $s=x$. Then to prove (5) it is enough to show that $u, v$ are connected in $H-\{x, t\}$ when $t \neq u$ and $t \neq v$. This is evident if $C_{2}$ is a tree or $t \notin \mu_{2}$. So let $t \in \mu_{2}$ and $C_{2}$ be not a tree. If $A(u) \cap$ $A(v) \neq \varnothing$, there is a chain connecting $u$, $v$ in $H-\{x, t\}$. So we take without loss of generality $A(u)=x_{j}$ and $A(v)=x_{i}$. If now $x_{j} \in A(y)$, then $u, v$ are connected through $x_{j}$ and $y$ in $H-\{x, t\}$. So we take $A(y)=x_{i}$. If $x_{j} \in A(x)$, then $y$ would not have been joined to $v$, so $A(x)=x_{i}$. Now in $G, x_{j}$ is connected to some vertex $z$ of $\mu_{1}$ by a chain with all intermediate vertices belonging to $C_{1}$ but not to $\mu_{1}$. Now we obtain a chain connecting $u, v$ in $H-\{x, t\}$ by going from $u$ to $x_{j}, x_{j}$ to $z, z$ to $y$ along $\mu_{1}, y$ to $x_{i}$, and $x_{i}$ to $v$. Thus we may take $\{s, t\} \cap\left\{x_{i}, x_{j}, x, y\right\}=\varnothing$.

Next let $s=u$. If $t \notin \mu_{1}$, then (5) is trivial, so let $t \in \mu_{1}$. Suppose first that $C_{2}$ is a tree. Then we obtain a chain connecting $x, y$ in $H-\{u, t\}$ by going from $x$ to $x_{i}$ or $x_{j}$, then to $v$ through a suitable pendant vertex of $C_{2}$ and then to $y$. If $C_{2}$ is not a tree, the situation is similar to that of the preceding paragraph. Thus we take $\{s, t\} \cap\left\{x_{i}, x_{j}, x, y, u, v\right\}=\varnothing$.

If none of $s, t$ belongs to $\mu_{1}$, then (5) is trivial. So let $s \in \mu_{1}$.

Suppose now that $C_{2}$ is a tree. Then for any fixed vertex $t$, there are chains in $H-\{s, t\}$ from one of $u, v$ to both $x_{i}$ and $x_{j}$, and a chain from the other (of the vertices $u, v$ ) to $x_{i}$ or $x_{j}$. Hence $u, v$ are connected and (5) follows.

Suppose next that $C_{2}$ is not a tree. Obviously we may take $s \in \mu_{1}$ and $t \in \mu_{2}$. If now $A(x) \cap A(y) \neq \varnothing$ or $A(u) \cap A(v) \neq \varnothing$, then again (5) follows. So we may take $A(x)=x_{i}, A(y)=x_{j}, A(u)=x_{j}$ $A(v)=x_{i}$. Now we obtain a chain connecting $x, y$ in $H-\{s, t\}$ by going from $x$ to $u, u$ to $x_{j}, x_{j}$ to $y$. This proves (5) completely.

Now by a repeated application of the above procedure we reduce the graph until finally $f(s, t)=1$ for any two vertices. The final graph has degrees $d_{1}, d_{2}, \cdots, d_{n}$ and is triconnected and this completes the proof of the theorem.

Perhaps necessary and sufficient conditions, similar to the conditions (1) to (4) above, for the existence of a $p$-connected graph with prescribed degrees $d_{1}, d_{2}, \cdots, d_{n}$ can be obtained for all $p \geqq 3$, but the authors have not yet succeeded in this. 


\section{REFERENCES}

1. M. D. Plummer, On minimal blocks, Trans. Amer. Math. Soc. 134 (1968), 85-94.

2. A. Ramachandra Rao, Some extremal problems and characterizations in the theory of graphs, a thesis submitted to the Indian Statistical Institute, Calcutta, 1969.

Received August 13, 1969.

Indian Statistical Institute, Calcutta 



\section{PACIFIC JOURNAL OF MATHEMATICS}

\section{EDITORS}

H. SAMELSON

Stanford University

Stanford, California 94305

\section{RichaRd PIERCe}

University of Washington

Seattle, Washington 98105
J. DUGUNDJI

Department of Mathematics

University of Southern California

Los Angeles, California 90007

RICHARD ARENS

University of California

Los Angeles, California 90024

\section{ASSOCIATE EDITORS}

E. F. BECKENBACH

B. H. NeUManN

F. WOLF

K. YosHIDA

\section{SUPPORTING INSTITUTIONS}

UNIVERSITY OF BRITISH COLUMBIA CALIFORNIA INSTITUTE OF TECHNOLOGY UNIVERSITY OF CALIFORNIA MONTANA STATE UNIVERSITY

UNIVERSITY OF NEVADA

NEW MEXICO STATE UNIVERSITY

OREGON STATE UNIVERSITY

UNIVERSITY OF OREGON

OSAKA UNIVERSITY

UNIVERSITY OF SOUTHERN CALIFORNIA
STANFORD UNIVERSITY

UNIVERSITY OF TOKYO

UNIVERSITY OF UTAH

WASHINGTON STATE UNIVERSITY

UNIVERSITY OF WASHINGTON

${ }^{*} \quad{ }^{*} \quad{ }^{*}$
AMERICAN MATHEMATICAL SOCIETY
CHEVRON RESEARCH CORPORATION
TRW SYSTEMS
NAVAL WEAPONS CENTER




\section{Pacific Journal of Mathematics}

\section{Vol. 33, No. $1 \quad$ March, 1970}

Mir Maswood Ali, On some extremal simplexes ................... 1

Silvio Aurora, On normed rings with monotone multiplication........... 15

Silvio Aurora, Normed fields which extend normed rings of integers....... 21

John Kelly Beem, Indefinite Minkowski spaces..................... 29

T. F. Bridgland, Trajectory integrals of set valued functions ........... 43

Robert Jay Buck, A generalized Hausdorff dimension for functions and sets ......................................... 69

Vlastimil B. Dlab, A characterization of perfect rings . . . . . . . . . . . . 79

Edward Richard Fadell, Some examples in fixed point theory ............ 89

Michael Benton Freeman, Tangential Cauchy-Riemann equations and uniform approximation ............................. 101

Barry J. Gardner, Torsion classes and pure subgroups ................ 109

Vinod B. Goyal, Bounds for the solution of a certain class of nonlinear

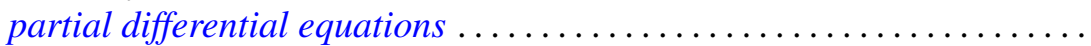

Fu Cheng Hsiang, On C, 1 summability factors of Fourier series at a given

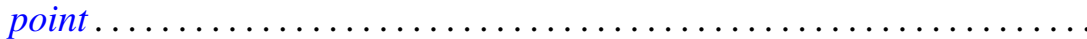

Lawrence Stanislaus Husch, Jr., Homotopy groups of PL-embedding

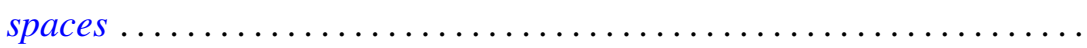

Daniel Ralph Lewis, Integration with respect to vector measures..........

Marion-Josephine Lim, $\mathscr{L}-2$ subspaces of Grassmann product spaces

Stephen J. Pierce, Orthogonal groups of positive definite multilinear functionals

W. J. Pugh and S. M. Shah, On the growth of entire functions of bounded index.

Siddani Bhaskara Rao and Ayyagari Ramachandra Rao, Existence of triconnected graphs with prescribed degrees . . .

Ralph Tyrrell Rockafellar, On the maximal monotonicity of subdifferential

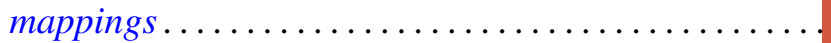

R. Shantaram, Convergence of a sequence of transformations of distribution functions. II ...............................

Julianne Souchek, Rings of analytic functions..............

Ted Joe Suffridge, The principle of subordination applied to functions of several variables...

Wei-lung Ting, On secondary characteristic classes in cobordism

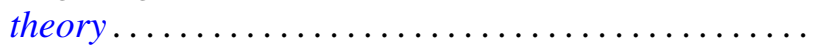

Pak-Ken Wong, Continuous complementors on $B^{*}$-algebras ...

Miyuki Yamada, On a regular semigroup in which the idempotents form a band. 